\title{
Valeur alimentaire des foins traités par l'ammoniac
}

\author{
J.P. DULPHY, P. ZWAENEPOEL *, A. KOMAR ** \\ et $S$. ABOULFARAJ \\ Avec la collaboration technique de Madeleine Dudilieu, \\ Jacqueline JAMOT, H. BousQuet et L. L'Hotelier \\ I.N.R.A., Laboratoire des Aliments, Theix, F 63122 Ceyrat \\ * C.E.M.A.G.R.E.F., Echelon de Montoldre, F 03150 Varennes-sur-Allier \\ ** Faculty of Animal Husbandry, Padjadjaran State University \\ Bukit Dago Utara Street, Bandung, West Java, Indonesie
}

\section{Résumé}

Afin de mesurer, avec des foins de valeurs très différentes, l'effet du traitement à l'ammoniac, nous avons préparé 6 foins traités avec 30 à $40 \mathrm{~kg}$ d'ammoniac anhydre par tonne et nous avons comparé leur composition chimique, leur valeur alimentaire et les caractéristiques de leur digestion ruminale chez le mouton à celle des foins avant traitement.

Le traitement augmente fortement la teneur en MAT des foins, qui passe de 86 à $152 \mathrm{~g} / \mathrm{kg}$ de MS (37 p. 100 de l'ammoniac ayant été fixé sur les foins). Ceci se traduit aussi par une augmentation des matières azotées non digestibles qui passent en moyenne de 44 à $67 \mathrm{~g} / \mathrm{kg}$ de MS, ce qui signifie qu'apparemment seulement $64 \mathrm{p} .100$ de l'ammoniac fixé a été utilisé par les animaux.

Par ailleurs, l'ingestibilité et la digestibilité des foins ont été augmentées nettement pour les foins de faible valeur alimentaire, mais non pour ceux qui avaient une valeur élevée.

Seul le comportement des animaux recevant un foin de valeur élevée avant traitement a été modifié par le traitement : baisse de la durée des grands repas et de la durée de rumination.

Le traitement a eu peu d'effet sur le pH du contenu du rumen, mais a fait passer en moyenne la teneur en $\mathrm{NH}_{3}$ du jus de rumen de 40 à $170 \mathrm{mg} / 1$. Il a aussi, et curieusement, fait baisser son activité cellulolytique.

Ces résultats sont discutés. La nécessité de mieux préciser le devenir de l'ammoniac fixé est souligné, ainsi que celle de préciser la signification de la baisse d'activité cellulolytique dans le rumen induite par le traitement.

Mots clés : foin, traitement à l'ammoniac, ruminant, digestibilité, ingestibilité.

\section{Introduction}

Le traitement des fourrages pauvres à l'ammoniac est connu depuis longtemps (Nikolaeva, 1938), mais ne s'est développé que récemment (ZAFren, 1959 ; BERGNER 
\& Marienburg, 1971 ; Chomyszyn \& Ziolecica, 1972 ; Sundstol, Coxworth \& Mowar, 1978). Il est très intéressant pour les pailles car il associe les propriétés basiques de l'ammoniac (rupture des liaisons hemicelluloses-lignine) à un apport d'azote non protéique susceptible d'être utilisé ensuite par les bactéries du rumen et compensant la carence en azote des fourrages pauvres.

Pour les foins, ce traitement est étudié depuis quelques années, aussi bien pour la conservation des foins humides que pour l'amélioration, en général, de la valeur alimentaire des foins, correctement séchés ou non, mais ayant une faible valeur (KNAPP, Holt \& Lechtenberg, 1975 ; Lechtenberg et al., 1977 ; UtLey, McCormick et McCullough, 1978; Buettner, 1978 ; Horn et al., 1983 ; Stallcup et al., 1982). Les résultats sont fort encourageants au niveau de la conservation, quoique irréguliers selon l'humidité du fourrage et les conditions de traitement. Pour une dizaine de foins étudiés les teneurs en matières azotées $(\mathrm{N} \times 6,25)$ sont passées de 95 à $172 \mathrm{~g} / \mathrm{kg}$ de MS. L'augmentation est plus importante lorsque le foin traité est plus humide et la dose d'ammoniac plus élevée (MoOre et al., 1981). Pour ces foins, l'augmentation de la digestibilité in vitro a été en moyenne de 10 points, mais peu de mesures ont été faites in vivo (Buettner et al., 1982 ; Stallcup et al., 1982 ; Utley, McCormick \& McCullough, 1978). Enfin, les variations d'ingestibilité n'ont pratiquement pas été étudiées (BuetTner et al., 1982) ni, de même, les caractéristiques de la digestion de ces foins dans le rumen. C'est pourquoi, nous nous sommes intéressés à l'intérêt de cette technique pour améliorer la valeur alimentaire des foins, en particulier ceux récoltés tardivement.

Nous avons donc traité 6 foins correctement séchés qui ont ensuite été distribués à des moutons. Pour 3 de ces foins les manifestations du comportement alimentaire des animaux ont été enregistrées, ainsi que certaines caractéristiques de la digestion dans le rumen.

\section{Matériel et méthodes}

\section{A. Fourrages}

Nous avons utilisé 6 foins de valeurs très différentes dont les caractéristiques sont rapportées dans le tableau 1. Ces 6 foins ont été pour partic traités en tas. Chaque tas était constitué de 300 à $500 \mathrm{~kg}$ environ de foin conditionné en balles de moyenne densité et était enveloppé le plus hermétiquement possible par une bâche plastique. Le traitement a été fait selon la méthode norvégienne de SundsTó, Coxworth \& Mowat (1978), l'ammoniac étant versé par un tuyau dans un récipient placé dans le tas, à sa base. La dose d'ammoniac utilisée a été de 3 p. 100 (sauf pour la fléole : 4 p. 100). Les traitements ont été effectués soit en août 1981, soit en avril 1982 et les foins n'ont été découverts qu'au bout de 2 mois pour être distribués à des moutons.

Les foins, traités ou non, ont été distribués hachés en brins de 2 à $4 \mathrm{~cm}$. 
Tableau 1

Caractéristiques et composition chimique des foins avant et après traitement. Chemical composition of hay before and after treatment.

\begin{tabular}{|c|c|c|c|c|c|}
\hline \multirow{2}{*}{$\begin{array}{l}\text { Espèce végétale } \\
\text { Species }\end{array}$} & \multirow{2}{*}{$\begin{array}{c}\text { Stade de } \\
\text { récolte } \\
\text { Harvesting } \\
\text { time }\end{array}$} & \multirow{2}{*}{$\begin{array}{c}\text { Traitement } \\
\text { Treatment }\end{array}$} & \multicolumn{3}{|c|}{$\begin{array}{r}\text { Teneurs en } \mathrm{g} / 1000 \mathrm{~g} \text { de } \mathrm{MS} \\
\text { Levels in } \mathrm{g} / \mathrm{kg} \mathrm{DM}\end{array}$} \\
\hline & & & $\begin{array}{l}\text { Cendres } \\
\text { Ashes }\end{array}$ & $\begin{array}{c}\text { Matières } \\
\text { azotées totales } \\
\text { Crude protein }\end{array}$ & $\begin{array}{l}\text { Cellulose } \\
\text { brute } \\
\text { Crude fibre }\end{array}$ \\
\hline \multirow{2}{*}{$\begin{array}{l}\text { Ray-grass d'Italie (1) } \\
\text { Italian Rye Grass }\end{array}$} & \multirow{2}{*}{$\begin{array}{l}\text { Tardive } \\
\text { Late }\end{array}$} & - & 93 & 46 & 382 \\
\hline & & + & 94 & 91 & 371 \\
\hline \multirow{2}{*}{$\begin{array}{l}\text { Ray-grass d'Italie (2) } \\
\text { Italian Rye Grass }\end{array}$} & \multirow{2}{*}{$\begin{array}{l}\text { Moyen } \\
\text { Mid }\end{array}$} & - & 58 & 61 & 349 \\
\hline & & + & 56 & 128 & 352 \\
\hline \multirow{2}{*}{$\begin{array}{l}\text { Ray-grass d'Italie ( } 3 \text { ) } \\
\text { Italian Rye Grass }\end{array}$} & \multirow{2}{*}{$\begin{array}{l}\text { Précoce } \\
\text { Early }\end{array}$} & - & 66 & 84 & 249 \\
\hline & & + & 64 & 157 & 262 \\
\hline \multirow{2}{*}{$\begin{array}{l}\text { Fiéole (4) } \\
\text { Timothy }\end{array}$} & \multirow{2}{*}{$\begin{array}{l}\text { Tardive } \\
\text { Late }\end{array}$} & - & 46 & 90 & 358 \\
\hline & & + & 45 & 177 & 364 \\
\hline \multirow{2}{*}{$\begin{array}{l}\text { Luzerne (5) } \\
\text { Lucerne }\end{array}$} & \multirow{2}{*}{$\begin{array}{l}\text { Tardive } \\
\text { Late }\end{array}$} & - & 102 & 142 & 386 \\
\hline & & + & 126 & 226 & 329 \\
\hline \multirow{2}{*}{$\begin{array}{l}\text { Fétuque (6) } \\
\text { Tall Fescue }\end{array}$} & \multirow{2}{*}{$\begin{array}{l}\text { Tardive } \\
\text { Late }\end{array}$} & - & 99 & 90 & 304 \\
\hline & & + & 100 & 135 & 315 \\
\hline
\end{tabular}

B. Animaux

Nous avons utilisé des moutons (béliers castrés de race Texel) regroupés en lots de 6 et pesant environ $70 \mathrm{~kg}$. Ces moutons étaient placés en cage à métabolisme. En outre, des moutons porteurs d'une fistule du rumen ( 2 par lot) ont également reçu les foins de ray-grass.

\section{Schéma expérimental}

Tous les foins ont été distribués à volonté pendant 3 semaines, les 2 premières servant à adapter les animaux à leur régime et la $3^{\circ}$ à mesurer exactement les quantités de fourrage offertes, refusées et excrétées. Chaque foin a été distribué en 2 repas $(8 \mathrm{~h}$ et $16 \mathrm{~h})$ et les refus, enlevés le lendemain matin, étaient tolérés à 10 p. 100 environ des quantités offertes.

Chacun des foins de ray-grass non traités a été distribué simultanément avec son homologue traité au cours d'une première période, respectivement à 3 moutons d'un lot, puis les foins ont été inversés au cours d'une seconde période de contrôle. 
Les autres foins ont été distribués successivement, sans inversion, à un lot de 6 moutons, d'abord non traités, puis traités.

De même les animaux fistulisés, recevant les foins de ray-grass, ont d'abord reçu les foins non traités, puis traités.

\section{Mesures}

Nous avons mesuré la digestibilité et l'ingestibilité des 12 fourages étudiés avec les animaux maintenus en cage à métabolisme.

Par ailleurs, les manifestations du comportement alimentaire et mérycique des moutons non fistulisés ont été enregistrées, pour les foins de ray-grass, lors des périodes de mesure, selon la méthode de Ruckebusch (1963).

Chez les moutons fistulés, nous avons prélevé du jus de rumen durant 2 jours consécutifs $0,1,2,3,4,6$ et 8 h après la distribution du repas du matin.

En outre, nous avons estimé l'activité cellulolytique du jus de rumen des moutons fistulisés du rumen en mesurant la vitesse de digestion d'une même paille broyée, placée dans des sachets de nylon suspendus dans le rumen des moutons fistulisés et retirés au bout de $8,24,48$ et 72 h après leur mise en place (CHENOST et al., 1970). Selon la même méthode, la vitesse de digestion des foins de ray-grass a été mesurée chez d'autres moutons fistulisés recevant un bon foin de luzcrne.

\section{E. Analyses}

Les échantillons représentatifs des aliments offerts (par lot), refusés (par lot) et des fèces (par animal) ont été déshydratés chaque jour dans une étuve ventilée à $80^{\circ} \mathrm{C}$ pendant 24 heures. Un seul échantillon de chaque fourrage offert, un des refus et un des fèces ont été conservés. Ces échantillons finaux ont été analysés pour déterminer leur teneur en cendres par incinération, en matières azotées totales $($ MAT, $\mathrm{N} \times 6,25$ ) selon la méthode de Kjeldahl et cn cellulose brute selon la méthode de Weende.

La teneur en MAT des foins traités a été celle déterminée sur des échantillons broyés, mais non passés à l'étuve.

L'analyse des caractéristiques des jus de rumen a été cffectuée selon les modalités décrites par ailleurs (DulPHy et al., 1983) mais seulement pour le pH et la teneur en ammoniac.

\section{Résultats}

\section{A. Composition chimique}

La composition chimique des foins après traitement est donnée dans le tableau 1. Le traitement à l'ammoniac a fait passer la teneur en MAT de 86 à $152 \mathrm{~g} / \mathrm{kg}$ de MS, soit une augmentation moyenne de $77 \mathrm{p} .100$ correspondant à une fixation de $13 \mathrm{~g}$ d'ammoniac par $\mathrm{kg}$ de MS. Cette fixation (37 p. 100 en moyenne) 
semble d'autant plus importante que la teneur en azote initiale du foin est élevée (fig. 1). Les teneurs en matières minérales et en cellulose brute n'ont pas, en moyenne, été modifiées par le traitement.

\section{B. Ingestibilité}

L'ingestibilité des foins est donnée dans le tableau 2. Elle a été augmentée par le traitement de 2 à 28 p. 100, les augmentations les plus fortes étant enregistrées pour les foins les moins bien ingérés avant traitement (fig. 2) : + 18 p. 100 pour les 4 foins les moins ingestibles.

TABleau 2

Quantités ingérées et digestibilité des foins avant et après traitement (6 jours de mesure). Intake and digestibility of hay before and after treatment (6 days of measurements).

\begin{tabular}{|c|c|c|c|c|c|}
\hline & \multirow{2}{*}{$\begin{array}{c}\text { Quantités } \\
\text { ingérées de MS } \\
\text { DM Intake } \\
\mathrm{g} / \mathrm{kg} \mathrm{P0.75} \\
\mathrm{g} / \mathrm{kg} W^{0.75}\end{array}$} & \multicolumn{3}{|c|}{$\begin{array}{l}\text { Digestibilité \% } \\
\text { Digestibility }\end{array}$} & \multirow{2}{*}{$\begin{array}{c}\text { Teneur en } \\
\text { matières azotées } \\
\text { non digestibles } \\
\\
\text { Undigestible } \\
\text { crude protein } \\
\text { (g/kg MS) } \\
(g / k g \text { DM) }\end{array}$} \\
\hline & & $\begin{array}{c}\text { Matière } \\
\text { organique } \\
\text { Organic } \\
\text { matter }\end{array}$ & $\begin{array}{l}\text { Matières } \\
\text { azotées } \\
\text { totales } \\
\text { Crude } \\
\text { protein }\end{array}$ & $\begin{array}{c}\text { Cellulose } \\
\text { brute } \\
\text { Crude fibre }\end{array}$ & \\
\hline $\begin{array}{l}\text { (1) Témoin } \\
\text { Untreated }\end{array}$ & $34,0 \pm 5,2^{a}$ & $50,7^{\mathfrak{a}}$ & 27,8 & 55,4 & 36 \\
\hline $\begin{array}{l}\text { Tra:té } \\
\text { Trcated }\end{array}$ & $41,6 \pm 10,2^{a}$ & $59,3^{\prime \prime}$ & 47,1 & 64,3 & 48 \\
\hline $\begin{array}{l}\text { (2) Témoin } \\
\text { Untrcated }\end{array}$ & $48,0 \pm 4,2^{a}$ & $57,0: a$ & 39,0 & 57,7 & 37 \\
\hline $\begin{array}{l}\text { Traité } \\
\text { Treated }\end{array}$ & $52,8 \pm 7,6^{a}$ & $59,1^{a}$ & 49,2 & 65,9 & 65 \\
\hline $\begin{array}{l}\text { (3) T'émoin } \\
\text { Untreated }\end{array}$ & $62,6 \pm 5,6^{a}$ & $70,5^{a}$ & 55,6 & 61,9 & 37 \\
\hline $\begin{array}{l}\text { Traité } \\
\text { Treated }\end{array}$ & $64,2 \pm 5,8^{a}$ & $68,2^{\prime \prime}$ & 50,9 & 69,9 & 77 \\
\hline $\begin{array}{l}\text { (4) Témoin } \\
\text { Untreated }\end{array}$ & $44.2 \pm 4,4^{a}$ & $58,1^{a}$ & 44,4 & 65,8 & 50 \\
\hline $\begin{array}{l}\text { Traité } \\
\text { Treated }\end{array}$ & $56,4 \pm 3,2^{b}$ & $64,3^{\prime \prime}$ & 55,8 & 76,6 & 78 \\
\hline $\begin{array}{l}\text { (5) Témoin } \\
\text { Intrated }\end{array}$ & $48,5 \pm 4,3 a$ & $51,0^{a}$ & 59,9 & 47,6 & 57 \\
\hline $\begin{array}{l}\text { Traité } \\
\text { Treated }\end{array}$ & $55,4 \pm 6,8^{a}$ & $55,7^{\prime \prime}$ & 69,5 & 46,8 & 69 \\
\hline $\begin{array}{l}\text { (6) Témoin } \\
\text { Untreated }\end{array}$ & $57,6 \pm 4,1 \mathrm{u}$ & $55,1^{a}$ & 50,7 & 58,0 & 44 \\
\hline $\begin{array}{l}\text { Traité } \\
\text { Treated }\end{array}$ & $69,2 \pm 2,2^{a}$ & $61,8^{b}$ & 51,0 & 73,3 & 66 \\
\hline
\end{tabular}



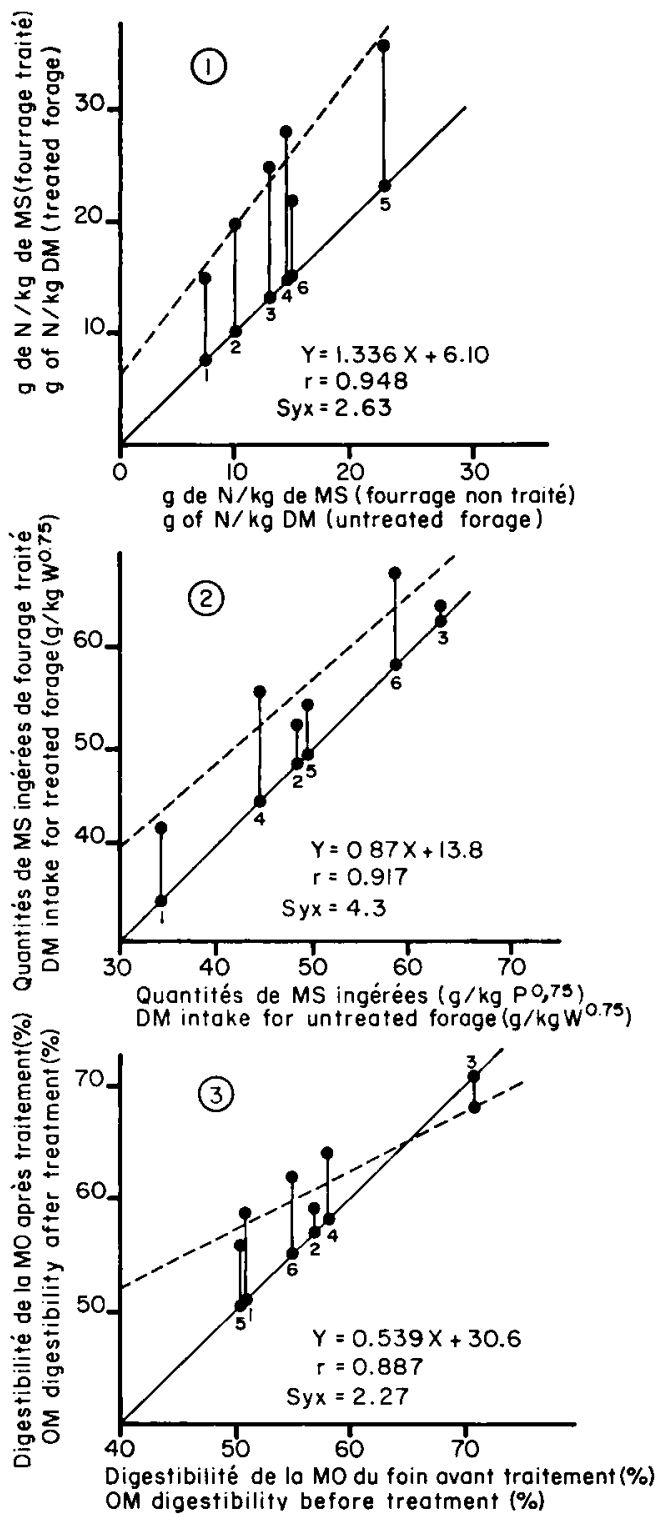

Fig. 1

Relation entre la teneur en azote des foins après le traitement et celle des foins non traités.

Relation between the level of nitrogen in hay before and after treatment.

Fig. 2

Relation entre les quantités de foin ingérées après traitement et celles ingérées avant traitement.

Relation between intake of hay before and after treatment.

FIG. 3

Relation entre la digestibilité de la matière organique des foins traités et celle des foins non traités.

Relation between O.M. digestibility of hay before and after treatment. foins traités - treated hay.

foins non trajtés - untreated hay. 


\section{Digestibilité de la matière organique et de la cellulose brute}

Les digestibilités de la matière organique et de la cellulose brute ont été augmentées par le traitement respectivement jusqu'à un maximum de $+8,6$ et $+19,3$ points pour le foin le moins digestible ( $\left.n^{0} 1\right)$. Par contre, il n'y a pas eu d'augmentation pour un foin de digestibilité correcte $\left(n^{\circ} 2\right)$ et même une diminution pour un foin de digestibilité élevée $\left(n^{\circ} 3\right)$.

Finalement, pour les 4 foins récoltés à un stade tardif $(1,4,5,6)$ l'augmentation de la digestibilité de la matière organique a été de 6,6 points.

\section{Digestibilité des matières azotées}

Le traitement à l'ammoniac a augmenté la digestibilité apparente des matières azotées, mais de façon moins importante que ne l'avait laissé espérer l'augmentation de la teneur en MAT. Il s'est traduit en effet par une augmentation importante (de 43,5 à $67,2 \mathrm{~g} / \mathrm{kg}$ de $\mathrm{MS}$, soit $+23,7 \mathrm{~g}$ ) de la teneur en matières azotées non digestibles. Il en résulte une augmentation de la teneur en matières azotées digestibles de $42,3 \mathrm{~g} / \mathrm{kg}$ de $\mathrm{MS}$, ce qui correspond à une digestion apparente de 64 p. 100 de l'ammoniac fixé sur le foin, soit 24 p. 100 de l'ammoniac utilisé pour le traitement).

\section{E. Comportement alimentaire et mérycique}

(tableau 3)

En moyenne le traitement à l'ammoniac n'a pas fait baissel les durées d'ingestion et de rumination (respectivement $-2,3$ et $-4,7$ p. 100). Par contre les durées unitaires d'ingestion et de rumination ont diminué (respectivement de $-12,1$ et de $-13,3$ p. 100) pour une augmentation moyenne des quantités ingérées de 7,7 p. 100 .

Malgré une augmentation de la quantité ingérée par grands repas et par jour et une baisse des durées unitaires d'ingestion et de rumination, le traitement à l'ammoniac n'a pas modifié significativement le comportement des animaux recevant le foin 1.

Pour le foin 2, les écarts enregistrés ont été très faibles à tous les niveaux, sauf une baisse légère, non significative, de la durée unitaire de mastication.

Pour le foin 3, par contre, le traitement a entrâné une baisse significative de la durée des grands repas et de la durée de rumination, ainsi qu'une baisse, non significative de la durée journalière d'ingestion et de la quantité ingérée par grands repas. Les durées unitaires d'ingestion et de rumination ont été légèrement et non significativement diminuées.

1. $p H$ (fig. 4)

\section{F. Caractéristiques du jus de rumen}

Le $\mathrm{pH}$ du jus de rumen a varié globalement entre 6,09 et 7,01 après distribution des foins non traités et de 6,49 et 7,00 pour les foins traités. Le pH moyen au cours de la journée n'a varié que légèrement pour les foins traités : $-0,05$ pour le foin $1(\mathrm{NS}),+0,11$ pour le foin $2(\mathrm{~S}),+0,04$ pour le foin 3 (NS). 


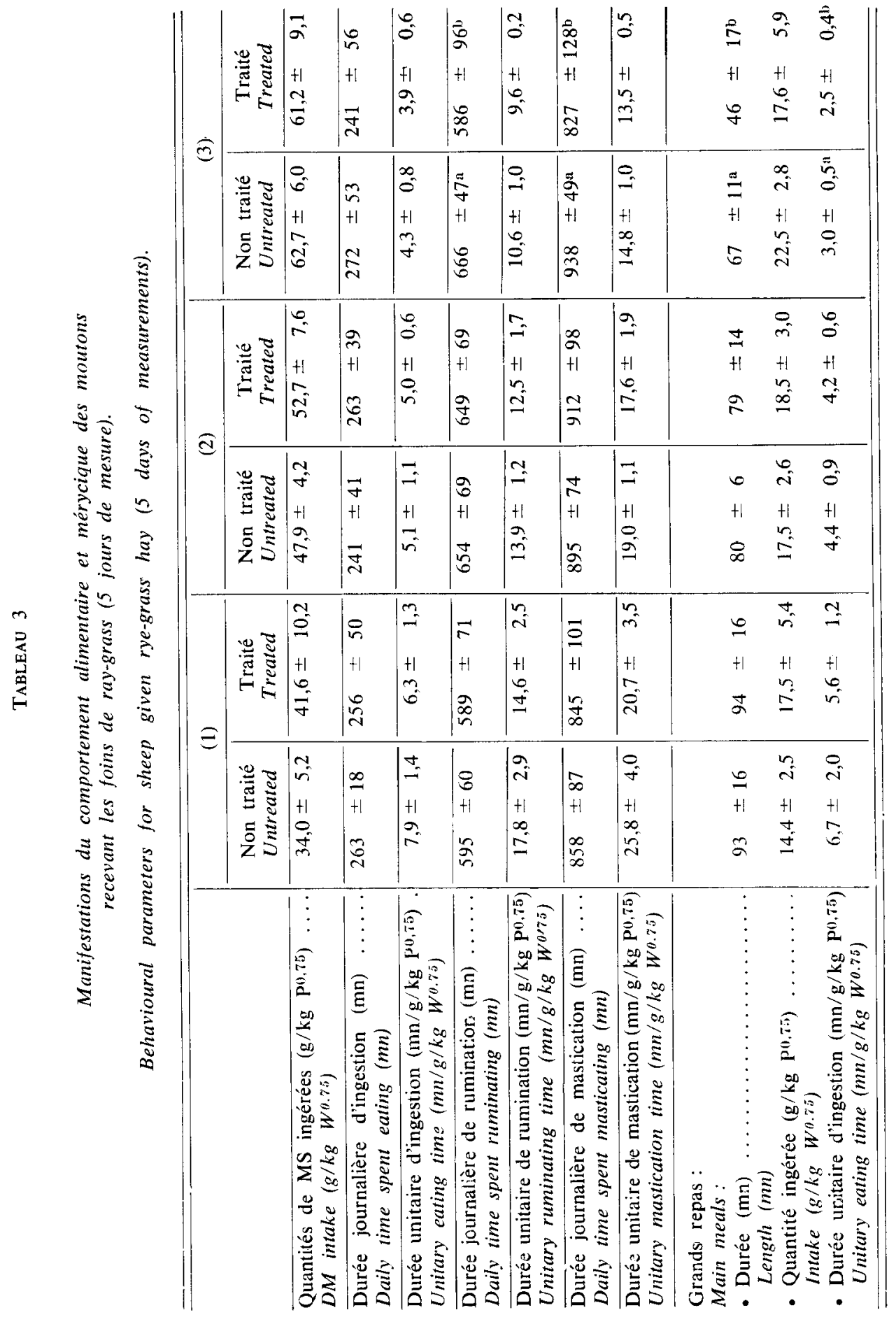



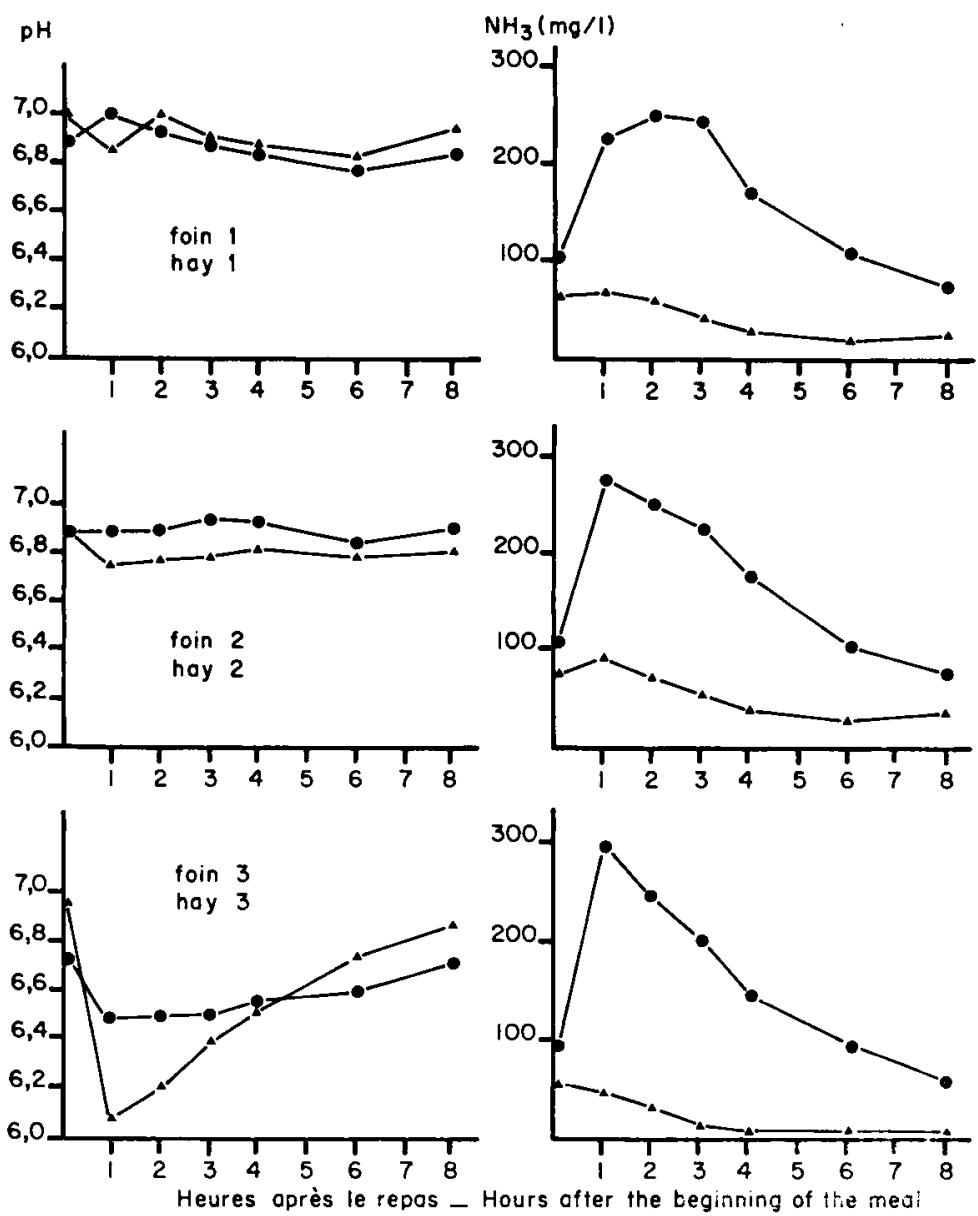

Fig. 4

FIG. 5

F1G. 4

Influence du traitement sur le $p H$ du jus de rumen. Effect of treatment on rumen $\mathrm{pH}$.

Fig. 5

Influence du traitement sur la teneur en $N H$, du jus de rumen. Effect of treatment on ammonia in rumen.

- foins non traités - untreated hay.

- foins traités - treated hay. 


\section{Teneur en $\mathrm{NH}_{3}$ (fig. 5)}

La teneur en ammoniac du jus de rumen a varié entre 6 et $90 \mathrm{mg} / 1$ pour les foins non traités et de 61 à 300 pour les foins traités. En moyenne, au cours de la journée, cette teneur est passée de $40 \mathrm{mg} / 1$ pour les foins non traités à $170 \mathrm{mg}$ pour les foins traités (différence hautement significative).

\section{Activité cellulolytique du jus de rumen (fig. 6)}

La vitesse de digestion de la paille jusqu'à 24 heures n'a pas été influencée par le traitement du foin distribué aux moutons. Par contre, entre 24 et 72 h la paille a été digérée plus rapidement en présence des foins non traités. Il y aurait donc, globalement, une baisse de l'activité cellulolytique due au traitement.
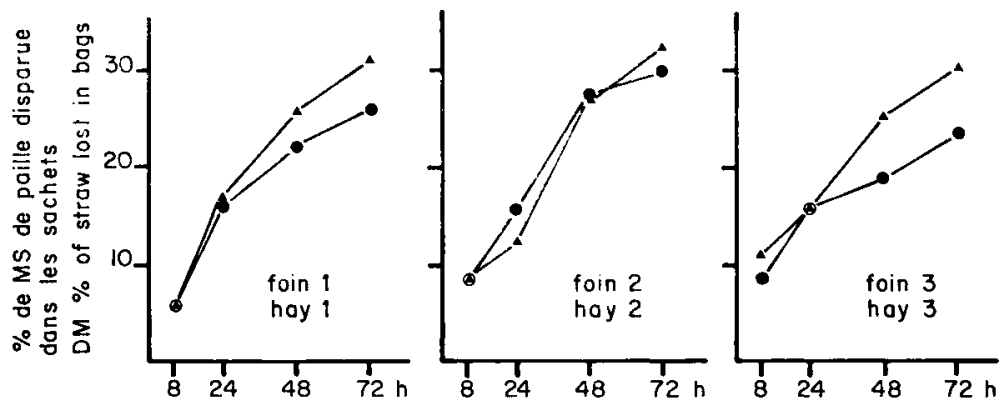

FIG. 6

Activité cellulolytique induite par les différents foins dans le rumen.

Cellulolytic activity caused by the different types of rye-grass hay in the rumen.

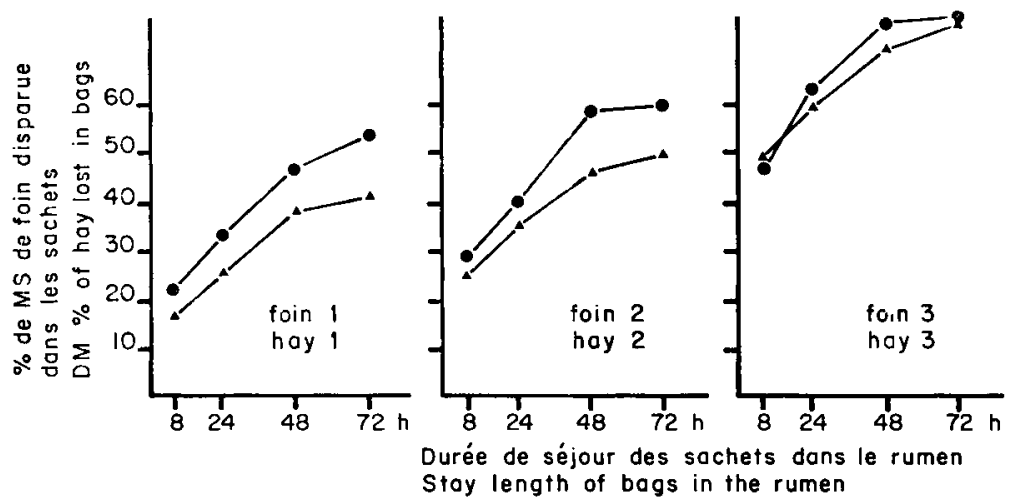

FIG. 7

Vitesse de digestion des foins dans le rumen de moutons recevant an foin de luzerne. Rate of digestion of rye-grass hay in the rumen of sheep given lacerne hay.

A foins non traités - untreated hay.

- foins traités - treated hay. 


\section{G. Vitesse de digestion des foins (fig. 7)}

La vitesse de digestion des foins a été nettement améliorée par le traitement, surtout pour le foin le moins digestible, mais très peu pour le foin 3 , récolté très précocément. En moyenne, la quantité de matière sèche disparue en $48 \mathrm{~h}$ dans les sachets est passée, pour les 3 foins de ray-grass de 53 p. 100 avant traitement à 61 p. 100 après traitement.

\section{Discussion - Conclusion}

Le traitement à l'ammoniac a augmenté nettement la teneur en azote des foins. L'augmentation que nous avons constatée est comparable à celle rapportée dans la bibliographie, même si elle est parfois très légèrement inférieure du fait que les foins que nous avons utilisés étaient secs. Au-delà de ce fait le principal problème est celui du devenir de l'azote ainsi fixé. YaHARA \& Numakawa (1978) signalent qu'une partie de l'ammoniac est faiblement fixée sur le fourrage traité, mais qu'une autre partie ( 33 p. 100 dans leur essai) l'est très fortement. C'est ce qui ressort aussi de nos mesures puisque la teneur en matières azotées non digestibles (MAND) est augmentée de $24 \mathrm{~g}$ après traitement (ce qui équivaut à $36 \mathrm{p} .100$ de l'ammoniac fixé par les foins). Dans l'essai de BuEtTNer et al. (1982) on assiste aussi à une élévation très importante de l'azote excrété dans les fèces puisque la teneur en MAND du foin étudié passe de $41 \mathrm{~g}$ avant traitement à $90 \mathrm{~g} / \mathrm{kg}$ de MS après ! Ces augmentations des teneurs en MAND peuvent résulter — soit de la présence d'azote ammoniacal solidement fixé sur les parois non digestibles et, par-là, non libéré dans le rumen - soit (et ou) d'une fermentation plus importante dans le gros intestin explicable par une baisse de l'activité cellulolytique dans le rumen; - soit (et ou) d'une augmentation de l'azote protéique insoluble au cours des réactions exothermiques de l'ammoniac (effet Maillard). Il pourrait aussi y avoir une meilleure utilisation de l'azote initial des foins par suite de la modification de la structure des glucides pariétaux. Nous n'avons pas suffisamment d'éléments pour discuter ces différentes hypothèses et bien que la première paraisse la plus vraisemblable, il est donc urgent d'approfondir l'étude de la valeur azotée réelle des foins traités à l'ammoniac. Nous abordons aussi ce problème avec des pailles traitées à l'ammoniac (Dulphy, Komar \& ZWAENEPOel, 1984).

Le traitement à l'ammoniac augmente aussi les quantités ingérées, en particulier pour les foins les moins digestibles. BuetTNer et al. (1982) signalent aussi, après traitement, une augmentation de 32 p. 100 de l'ingestibilité d'un foin chez des agneaux. Certains de nos foins (foins 1 et 2) étaient, manifestement, carencés en azote. Le traitement a donc pu joucr à la fois par un apport d'azote et par une augmentation de la vitesse de digestion du fourrage. Il est probable cependant que la carence en azote ait eu peu d'effet car les conséquences du traitement sur l'ingestibilité ont été indépendantes de la teneur en MAT des foins avant traitement. Par ailleurs, les activités cellulolytiques induites du jus de rumen des animaux recevant les 3 foins de ray-grass non traités ont été identiques, ce qui pourrait confirmer que la carence en azote n'a pas eu trop de conséquence sur l'ingestion de ces foins. 
L'observation concernant l'activité cellulolytique du jus de rumen lorsque les animaux reçoivent les fourrages traités est curieuse. Dulphy, Komar \& Zwaenepoel (1984) trouvent d'ailleurs la même chose avec une paille de blé. Nous avions déjà trouvé un effet dépressif de la soude sur cette activité cellulolytique (BIENAIME, 1979), mais cet effet n'a, à notre connaissance, jamais été signalé pour l'ammoniac qui, normalement, est un stimulant de l'activité microbienne. On pourrait penser à une évolution de la population microbienne vers une population à activité cellulolytique plus faible, mais en fait nous n'avons guère d'explication pour le moment.

L'effet du traitement sur la digestibilité de la matière organique est positif, mais assez faible dans nos essais. L'efficacité du traitement a pu être limitée par la faible humidité de nos foins, mais aussi par le fait que certains avaient déjà une digestibilité élevée. Pour les foins les moins digestibles les résultats sont d'ailleurs concordants avec ceux rapportés dans la bibliographie.

Les légères modifications du comportement alimentaire, surtout les baisses des durées unitaires d'ingestion et de rumination s'expliquent bien par l'augmentation de la digestibilité et de vitesse de digestion dues au traitement (Dulphy, Remond \& Theriez, 1979). Cependant, pour le foin de ray-grass le plus digestible (3) les animaux n'ont pas compensé une durée unitaire de mastication plus faible par une ingestion plus élevée.

Les caractéristiques de la digestion des foins traités à l'ammoniac n'ont pratiquement pas été étudiées. En ce qui concerne le pH, l'effet du traitement est négligeable, ce qui correspond à ce qui est connu pour les pailles (HorTon, 1978; Horton \& Steacy, 1979; Garrett et al., 1979). Il aurait pu même diminuer (Oji, Mowat \& Buchanan-Smith, 1979; Dulphy, Komar \& Zwaenipoel, 1984) s'il y avait eu une nette augmentation de la production des acides gras volatils, ce qui n'a pas dû être le cas pour nos foins de ray-grass. Les teneurs en ammoniac du jus de rumen n'ont pas été excessives, mais ont atteint des valeurs de $300 \mathrm{mg} / \mathrm{l}$, teneur cependant inférieure au seuil de toxicité. Ceci correspond aux résultats de Buettner et al. (1982) ou de Horton (1978) et Oit, Mowat \& Buchanan-Smith (1979) avec des pailles, mais n'est pas en accord avec les constatations de Cordesse \& Taba-Tabai (1981) et de Chomyszin \& Zrolecica (1972) qui indiquent une libération lente et progressive de l'ammoniac lié aux pailles traitées, probablement parce que ces dernières sont ingérées et digérées plus lentement.

En fin de compte nos essais confirment les rares résultats qui existent sul les foins traités à l'ammoniac. Le traitement a un effet positif plus important sur l'ingestion et la digestion des fourrages âgés et donc sur l'apport d'énergic nette et de matières azotées aux animaux. Il reste à préciser comment cette augmentation d'apport se traduit sur leurs performances, car seuls Uthey, McCormick \& McCullough (1978) ont effectué des essais dans ce sens. Les nôtres amènent en outre à se poser deux problèmes importants : celui du devenir de l'ammoniac fixé et celui de l'activité cellulolytique induite par les fourrages ainsi traités. Les éleveurs devront donc pour l'instant être prudents à propos du calcul de la valeur azotée de ces fourrages. 


\section{Summary}

Feeding value of ammonia-treated hays

Using hays of very different values, the effect of ammonia treatment was measured on 6 hays treated with $30-40 \mathrm{~kg}$ of anhydrous ammonia per ton. Their chemical composition, feeding value as well as the characteristics of their rumen digestion in sheep was compared to those of hays before treatment.

Ammonia treatment strongly increased the crude protein level of hays from 86 to $152 \mathrm{~g} / \mathrm{kg} \mathrm{DM}, 37 \mathrm{p} .100$ of $\mathrm{NH}_{3}$ being fixed during the treatment (table 1 and fig. 1). At the same time, the level of undigestible crude protein increased from 44 to $67 \mathrm{~g} / \mathrm{kg} \mathrm{DM}$ (table 2), 64 p. 100 only of the fixed ammonia being useful for the animals.

Intake and digestibility of hays increased very markedly for those with a low feeding value, but not for those with a good value (table 2, fig. 2, fig. 3).

The feeding behaviour of sheep receiving rye-grass hay was modified but only with hay of high value before treatment : decrease in the length of the main meals and in the time spent ruminating (table 3 ).

The effect of treatment on ruminal $\mathrm{pH}$ was small (fig. 4), but the level of ammonia in rumen juice increased from 40 to $170 \mathrm{mg} / \mathrm{l}$ (fig. 5). It was observed also a decrease in the cellulolytic activity of the rumen (fig. 6), when the rate of digestion was enhanced by the treatment (fig. 7).

Those results are discussed. A better understanding of the utilization of fixed ammonia is required so as to determine more accurately the meaning of the cellulolytic activity decrease in the rumen after treatment.

Key words : hay, ammonia treatment, ruminant, digestibility, feed intake.

Reçu en juillet 1983.

Accepté en décombre 1983.

\section{Références bibliographiques}

Bergner H., Marienburg J., 1971. Herstellung und Futterwert von amminisierten Roggenstrohpellets. Arch. Tierernähr., 21, 557-566.

Bienaime A., 1979. Facteurs de variation de la digestibilité des pailles de céréales. Thèse de Docteur de $3^{\circ}$ cycle. Université de Montpellier, 118 pages.

BUEtTNER M.R., 1978. Effect of ammoniation on the composition and digestion of forage fiber. Diss. Abstr. Inter., B, 39, 4134.

Buettner M.R., Lechtenberg V.L., Hendrix K.S., Hertel J.M., 1982. Composition and digestion of ammoniated tall fescue (Festuca arundinacea Schreb.) Hay. J. Anim. Sci., 54, 173-178.

Chenost M., Grenet E., Demarquilly C., Jarrige R., 1970. The use of the nylon bag technique for study of forage digestion in the rumen and for predicting feed value. Proc. XI Int. Grassld. Congress, 697-701.

Chomyszyn M., Zrolecica $A$., 1972. Utilization of ammoniated feeds in ruminant nutrition. In : Tracer studies on non protein for ruminants. IAEA. Vienna. FAO, 153-161.

Cordesse R., TABa-Tabai M., 1981. Alimentation d'agneaux à partir de la paille traitée à l'ammoniac. 2. Cinétique biochimique de la dégradation dans le rumen de la paille. Arn Zootech., 30, 299-312. 
Dulphy J.P., Breton J., Louyot J.M., Bienaime A., 1983. Etude de la valeur alimentaire des pailles de céréales traitées ou non à la soude. III. Influence du niveau d'apport d'aliment concentré. Ann. Zootech., 32, 53-80.

Dulphy J.P., Komar A., Zwaenepoel P., 1984. Effets comparés des traitements à l'ammoniac et à la soude sur la valeur alimentaire des fourrages pauvres (en préparation).

DUlPhy J.P., REMOND B., Theriez M., 1979. Ingestive behaviour and related activities in ruminants. Proc. Sth Int. Symposium on Ruminant Physiology. Y. Ruckebusch and P. Thivend, Ed. Clermont-Ferrand, 3-7 september, p. 103-122.

Garrett W.N., Wal.ker H.G., Kohler G.O., Hart M.R., 1979. Response of ruminants to diets containing sodium hydroxide or ammonia rice straw. J. Anim. Sci., 48, 92-103.

Horn G.W., Batchelder D.G., Manor G., Streeter C.L., Mc Laughlin G.L., 1983. Ammoniation of wheat straw and nature grass hay during baling of large round bales. Anim. Feed Sci. Technol., 8, 35-46.

Hor'TON G.M.J., 1978. The intake and digestibility of ammoniated cercal straw by cattle. J. Anim. Sci., 58, 471-478.

HorTon G.M.J., STEACY G.M., 1979. Effect of anhydrous ammonia treatment on the intake and digestibility of cereal straws by steers. J. Anim. Sci., 48, 1239-1249.

KNAPP W.R., Holt D.A., Lechtenberg V.L., 1975. Hay preservation and quality improvement by anhydrous ammonia treatment. Agron. J., 67, 766-769.

Lechtenberg V.L., Buettner M.R., Holt D.A., Richey C.B., Parsons S.D., 1977. Hay preservation by anhydrous ammonia treatment. Publ. : St Joseph, Michigan, U.S.A.; Am. Soc. Agric. Engin., 327-328, 338.

Moore K.J., Lechtenberg V.L., Johnson K.D., Hertel J.M., 1981. Ammoniation of mature grass hay at several moisture percentages (Abstr.). Agr. abst., 73rd annual meeting, Am. Soc. Agron. Purdue Univ. Publ. : Madison, Wisconsin, U.S.A., 134.

NikolaEva L.I., 1938. Ammonium hydroxyde treatment of straw. Problems Animal Husbandry (U.S.S.R.), 7, n' 3, 175-178. In : Chem. Abst., EPGE, 35, 817.

Oji U.I., Mowat D.N., Buchanan-Smith J.G., 1979. Nutritive value of thermoammoniated and steam treated maize stover : II. Rumen metabolites and rate of passage. Anim. Feed Sci. Techn., 4, 187-197.

RuCKEBUSCH Y., 1963. Recherches sur la régulation centrale du comportement alimentaire des ruminants. Thèse Doct. Sci. Nat., Université de Lyon. 213 p.

Stallcur O.T., Harrison K.L., Kreider D.L., Bayley P., 1982. Ammonia treatment of Bermudagrass hay. Arkansas Farm Res., 31, 1-9.

Sundstar F., Coxworth E., Mowat D.N., 1978. Improving the nutritive value of straw and other low quality roughages by treatment with ammonia. World Anim. Prod., 26, 13-21.

Utley P.R., Mc Cormick W.C., Mc Cullough M.E., 1978. Ammonia treatment of coastal Bermudagrass hay to improve digestibility. Ga. agric. Res., 20, 17-18.

Yahara N., Numakawa T,, 1978. Improvement of preservation and quality of semidricd hay by anhydrous ammonia treatment. Jap. J. Zoot. Sci., 49, 648-652.

ZAFREN S.J., 1959. Increasing the nutritive value of straw and at the same time adding digestible nitrogen. Dokl. Vses Akad. Sel'Skohoz. Nauk., 8, 9-14. In : Nutr. Abstr. Rev., 31, 252, 1961. 